\title{
Comprehensive search for intra- and inter-specific sequence polymorphisms among coding envelope genes of retroviral origin found in the human genome: genes and pseudogenes
} Nathalie de Parseval ${ }^{\dagger 1}$, Gora Diop ${ }^{\dagger 2}$, Sandra Blaise ${ }^{1}$, François Helle ${ }^{1}$, Alexandre Vasilescu ${ }^{2}$, Fumihiko Matsuda² and Thierry Heidmann*1

Address: ${ }^{1}$ UMR 8122 CNRS, Institut Gustave Roussy, 39 rue Camille Desmoulins, 94805 Villejuif Cedex, France and ${ }^{2}$ Centre National de Génotypage, 2, rue Gaston Crémieux, Évry, France

Email: Nathalie de Parseval - parseval@igr.fr; Gora Diop - diop@cng.fr; Sandra Blaise - sblaise@pasteur.fr; François Helle - francois.helle@ibl.fr; Alexandre Vasilescu - vasilesc@cng.fr; Fumihiko Matsuda - fumi@cng.fr; Thierry Heidmann* - heidmann@igr.fr

* Corresponding author †Equal contributors

Published: 09 September 2005

BMC Genomics 2005, 6:117 doi:10.1186/1471-2164-6-117

This article is available from: http://www.biomedcentral.com/147I-2164/6/117

(c) 2005 de Parseval et al; licensee BioMed Central Ltd.

This is an Open Access article distributed under the terms of the Creative Commons Attribution License (http://creativecommons.org/licenses/by/2.0), which permits unrestricted use, distribution, and reproduction in any medium, provided the original work is properly cited.

\begin{abstract}
Background: The human genome carries a high load of proviral-like sequences, called Human Endogenous Retroviruses (HERVs), which are the genomic traces of ancient infections by active retroviruses. These elements are in most cases defective, but open reading frames can still be found for the retroviral envelope gene, with sixteen such genes identified so far. Several of them are conserved during primate evolution, having possibly been co-opted by their host for a physiological role.
\end{abstract}

Results: To characterize further their status, we presently sequenced 12 of these genes from a panel of 91 Caucasian individuals. Genomic analyses reveal strong sequence conservation (only two non synonymous Single Nucleotide Polymorphisms [SNPs]) for the two HERV-W and HERV-FRD envelope genes, i.e. for the two genes specifically expressed in the placenta and possibly involved in syncytiotrophoblast formation. We further show - using an ex vivo fusion assay for each allelic form - that none of these SNPs impairs the fusogenic function. The other envelope proteins disclose variable polymorphisms, with the occurrence of a stop codon and/or frameshift for most - but not all - of them. Moreover, the sequence conservation analysis of the orthologous genes that can be found in primates shows that three env genes have been maintained in a fully coding state throughout evolution including envW and envFRD.

Conclusion: Altogether, the present study strongly suggests that some but not all envelope encoding sequences are bona fide genes. It also provides new tools to elucidate the possible role of endogenous envelope proteins as susceptibility factors in a number of pathologies where HERVs have been suspected to be involved.

\section{Background}

A large fraction ( $8 \%$ ) of the human genome contains elements of retroviral origin, with thousands of sequences closely related to the integrated proviral form of infectious retroviruses with the canonical $g a g, p r t, p o l$ and $e n v$ genes [1]. These elements, named human endogenous 
retroviruses (HERV), are most probably the proviral remnants of ancestral germline infections by active retroviruses, which have thereafter been transmitted in a Mendelian manner. HERVs have been grouped according to sequence homologies into more than 80 distinct families, each containing a few to several hundreds elements (reviewed in [2-4], see [5] for their classification). Most HERV genes are non-coding, due to either terminating mutations or deletions, but we have characterized 16 human endogenous env genes that have retained a coding capacity among the 30,000 endogenous proviral elements of the human genome [6]. The analysis of their transcriptome in healthy human tissues has revealed that three of them are highly expressed in the placenta, namely the erv3/HERV-R, the HERV-W and the HERV-FRD env genes [6].

Phylogenetic as well as functional analyses have revealed strong similarities between HERVs and the present-day infectious retroviruses, suggesting a common history and shared ancestors. Accordingly, it has been proposed that HERVs may still possess some of the functions of infectious retroviruses and as such have pathogenic effects, provided that they are transcriptionally active. Conversely, it is also plausible that HERV proteins may have been co-opted by the host for its benefit. Along this line, it has been proposed that the HERV envelope proteins could play a role in several processes including i) protection against infection by present-day retroviruses through receptor interference [7], ii) protection of the fetus against the maternal immune system via an immunosuppressive domain located in the envelope transmembrane (TM) subunit $[8,9]$, and iii) placenta morphogenesis through fusogenic effects allowing differentiation of cytotrophoblastic cells into the syncytiotrophoblast [10-12]. In accordance with a symbiotic role for HERVs, it has recently been shown that the HERV-W and HERV-FRD envelope gene products are highly fusogenic glycoproteins that are specifically expressed in the placenta and can mediate cellcell fusion ex vivo [12,13]. Involvement of HERV proteins in physiological processes, however, remains a debated issue, and definite evidence is still lacking. Because selection pressure on a functional gene should result in a limited mutation rate, the survey of single nucleotide polymorphisms (SNPs) among the human population is a way to evaluate functional constraints on these genes. Using this approach, we had previously demonstrated that one postulated candidate for a role in placentation, namely the highly-expressed erv3/HERV-R envelope gene carries a homozygous stop mutation resulting in a severe protein truncation in $1 \%$ of individuals of caucasian origin, which strongly suggests that it is not necessary for any fundamental placental function [14]. The unexpectedly low number of still coding envelope genes present in the human genome [6] now allows a comprehensive analysis of such genes to be performed, in order to assess their possible physiological and/or physiopathological role. Here, we analysed the SNP level of the 12 coding env genes present in the human genome that could be characterized by this approach, together with their conservation among the orthologous genes that can be identified in primates. The two series of data are consistent with a role beneficial to the host for some of the genes, whereas others are likely to be subjected to progressive inactivation. In both cases, the identified SNPs should be useful tools to evaluate the possible role of these genes as "susceptibility genes" in several human pathologies where HERVs have been suspected to be involved.

\section{Results \\ Structure and PCR-amplification of the fully coding HERV envelope genes}

Retroviral envelope genes are $2 \mathrm{~kb}-$ long sequences with no introns, that are located in the 3 ' domain of proviral elements (see Figure 1A for the genomic structure of a provirus and Figure $1 \mathrm{~B}$ and $1 \mathrm{C}$ for the description of the envelope gene and its product). Endogenous retroviruses being in most cases highly reiterated elements (see Table 1), the 3' PCR primers for env gene amplification had to be placed downstream of the provirus end to specifically amplify the family member of interest, whereas the $5^{\prime}$ primer was placed upstream of the env MET initiation codon (see Figure 1A). Among the 16 coding env genes that we had previously identified [6], 12 could be subjected to a systematic search for SNPs, including 3 out of the 6 HERV-K(HML-2) coding env genes (K1, K2, K4), 2 out of the 3 HERV-H coding env genes ( $\mathrm{H} 1$ and $\mathrm{H} 3)$, and the coding env genes of the $\mathrm{F}(\mathrm{c}) 1, \mathrm{~F}(\mathrm{c}) 2, \mathrm{~T}, \mathrm{~W}, \mathrm{R}, \mathrm{R}(\mathrm{b})$ and FRD families. Three HERV-K(HML-2) env genes could not be PCR amplified: the first one $(e n v \mathrm{~K} 3)$ is located in the centromeric region of chromosome 19, and its provirus is surrounded by stretches of repeated sequences, thus precluding the use of specific primers; the other two (env $\mathrm{K} 5$ and $e n v \mathrm{~K} 6$ ) belong to proviruses present only in a fraction of the caucasian population [15], with a low allele frequency ( 0.19 and 0.04 , respectively), thus precluding a statistically significant SNP study to be performed (unless a pre-selected population was used). It is noteworthy that proviruses K1, K2 and K4 have also been recently demonstrated to be polymorphic, since they exist in some individuals as a solo Long Terminal Repeat (LTR), devoid of internal sequences [16]. Yet, the allele frequencies of the complete provirus forms are high $(0.72,0.97$ and 0.89 , respectively), thus allowing an SNP study. Finally, sequence data for one of the 3 HERV-H coding env genes studied (i.e. envH2) yielded multiple sequence profiles (possibly due to the parasitic amplification of another member of the HERV-H family) and could not be analyzed further. The list of the 12 fully coding env genes that 

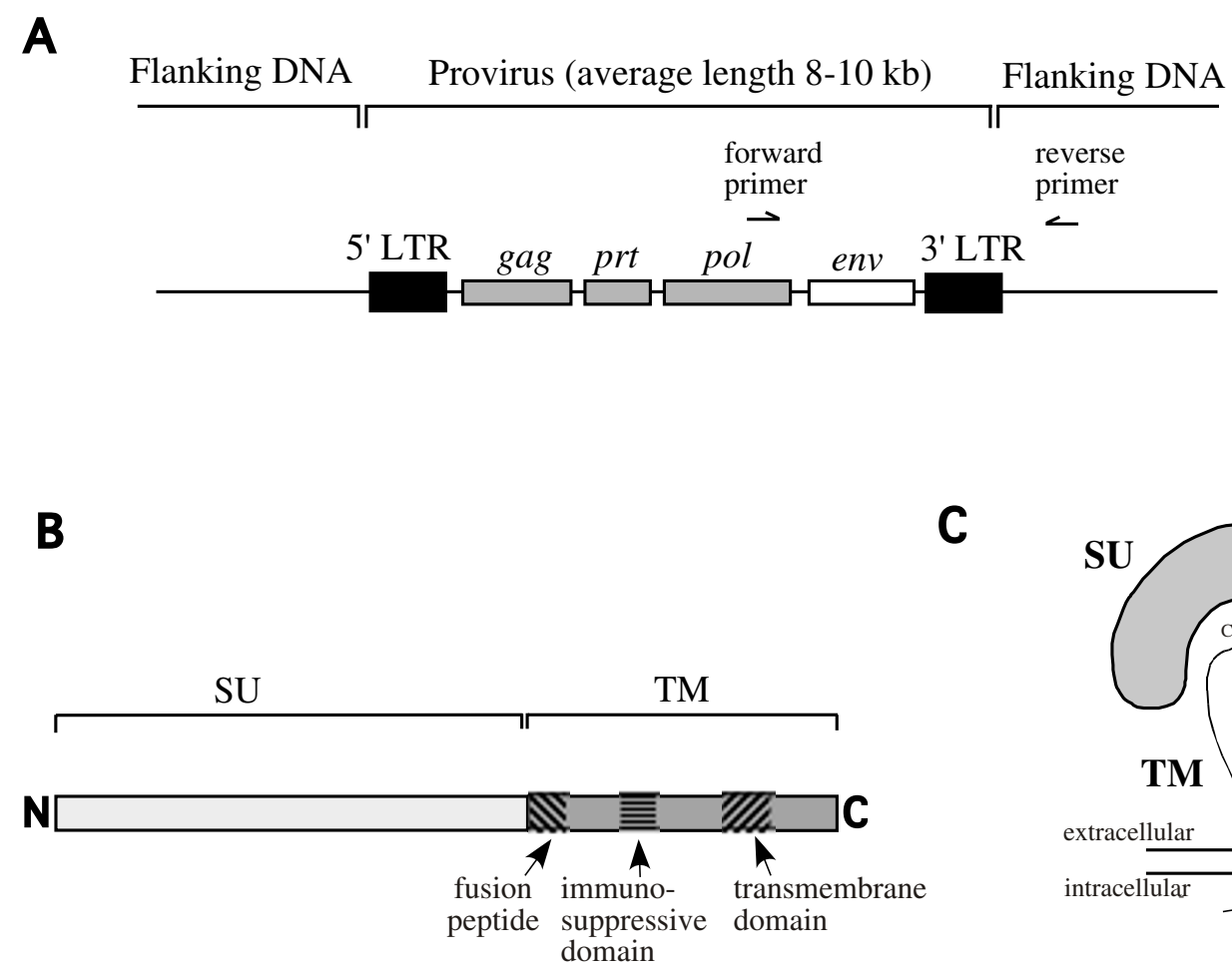

C

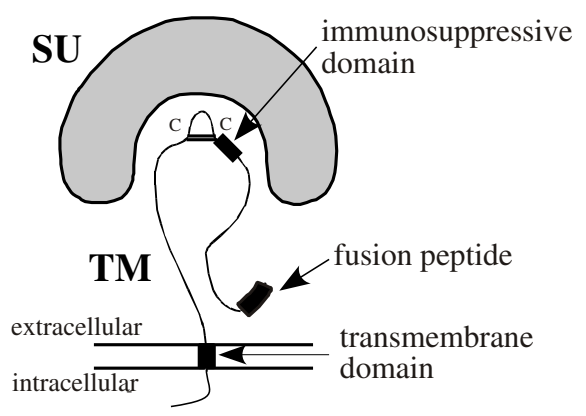

Figure I

Schematic representation of the proviral form of a retrovirus and its env gene products. A, Genomic structure of a provirus, with the four canonical retroviral genes gag (encoding the virion core proteins), prt (encoding the protease), pol (encoding the reverse transcriptase, RNAseH, integrase), and env (encoding the viral membrane protein). The position of the primers designed to amplify the coding env gene is indicated. B, Linear representation of a retroviral envelope protein and its functional domains. The env gene encodes a polypeptide which is cleaved into two proteins, the surface protein (SU), which is involved in receptor recognition, and the transmembrane subunit (TM), which anchors the whole envelope complex to the membrane and is directly responsible for cell membrane fusion and virus entry. The TM subunit contains three functional domains, the fusion peptide, the immunosuppressive domain and the transmembrane domain. C, Schematic structure of the envelope gene products. C-C: disulfide bound.

could finally be analyzed, together with the associated amplification primers used, is given in Table 1.

\section{SNP of the human coding envelope genes and haplotype analysis}

The 12 HERV coding env genes were PCR-amplified for 91 healthy Caucasian individuals, and each PCR product was directly sequenced without cloning. The identified SNPs are positioned on the protein sequences in Figure 2, and the number and nature of the variations (synonymous [i.e. silent] versus nonsynonymous [i.e. leading to an amino acid change] substitutions, stop or insertion/deletion [indel] mutations) are given in Figure 3. The complete list and detailed features - including frequencies - of the identified SNPs is available in additional data file 1. As illustrated in Figure 2 and 3, the number of SNPs for the 12 env genes varies significantly depending on the gene, but their distribution along the envelope sequences seems to be random. The number of SNPs per kb varies from 1 (for $e n v \mathrm{~W}$ ) to 10 (for $e n v \mathrm{~T}$ ). Studies on SNPs in intragenic regions of the human genome disclose average densities varying from 0.8 to 5 SNPs per $\mathrm{kb}[17,18]$. These studies also provide an estimate of the average ratio of synonymous versus non-synonymous SNPs, which is close to 1 in coding regions, whereas it is close to 0.5 in pseudogenes $[17,18]$. For the env genes, provided that the SNP number is high enough to validate this ratio (thus exclud- 
Table I: The coding envelope genes of the human genome studied for their SNPs.

\begin{tabular}{|c|c|c|c|c|c|}
\hline $\begin{array}{l}\text { Gene } \\
\text { name }\end{array}$ & $\begin{array}{l}\text { Bibliographic } \\
\text { gene name }\end{array}$ & $\begin{array}{l}\text { Family name (repbase } \\
\text { identifier) }\end{array}$ & $\begin{array}{l}\text { Approximate } \\
\text { number of } \\
\text { elements }\end{array}$ & Genomic localization & Amplification primers sequence $\left(5^{\prime}-3^{\prime}\right)$ \\
\hline envKI & - & $\begin{array}{l}\text { HERV-K(HML-2) } \\
\text { (HERVK) }\end{array}$ & $50-100$ & Chr I2: 5700843I-570I0527 (-) & $\begin{array}{l}\text { F: GGGAAATAGGGAAGGTGATA } \\
\text { R: ACATCCCTAACGCTTTAAG }\end{array}$ \\
\hline envK2 & $\begin{array}{l}\text { HML-2.HOM, } \\
\text { HERV-K } 108\end{array}$ & idem & idem & Chr7: 43673I7-43694I6 (-) & $\begin{array}{l}\text { F: GAGGTTTTGCTTGTGTTTCA } \\
\text { R: TTAGGCTTTCGGGACTTCAA }\end{array}$ \\
\hline envK4 & HERV-KI09 & idem & idem & Chr6: 78423 I 72-78425268 (-) & $\begin{array}{l}\text { F: GGGAAATAGGGAAGGTGATA } \\
\text { R: GGGTAGTATCAGTCGGGATG }\end{array}$ \\
\hline $\operatorname{env} F(c) I$ & - & HERV-F(c) I (-) & I & Chr:X: 95874| I8-95875872 (+) & $\begin{array}{l}\text { F: GCACCGACTCAGCACGAC } \\
\text { R: GCCTTGGCAACTAAACCATTC }\end{array}$ \\
\hline envF(c)2 & - & HERV-F(c)2 (-) & 15 & Chr7:I52498|67-I52499936 (-) & $\begin{array}{l}\text { F: GAAGGCACCTACACAACATC } \\
\text { R: GACACTTAATAGTTGCGACA }\end{array}$ \\
\hline envHI & $\mathrm{envH} / \mathrm{p} 62, \mathrm{HI} 9$ & HERV-H (HERVH) & 1000 & Chr2: 166767244-166768998 (-) & $\begin{array}{l}\text { F: ATGCCCTACTCTTGTTTACAC } \\
\text { R: AAATCTGGCAAACTACAAGC }\end{array}$ \\
\hline envH3 & envH/p59 & idem & idem & Chr2: I5593|277-I55932944 (+) & $\begin{array}{l}\text { F: TTTCTTCAAGCCATCACAGC } \\
\text { R: ACCCCATGTTCTAGTCTTCC }\end{array}$ \\
\hline envT & - & HERV-T (HERVS7I) & 50 & ChrI9: 2034|24I-20343I2I (+) & $\begin{array}{l}\text { F: TTGGATTCATCACTCCCA } \\
\text { R: CTGAAGGGAGTTCCTCCTAGG }\end{array}$ \\
\hline envW & Syncytin I & HERV-W (HERVI7) & 200 & Chr7: $91710108-91711724(-)$ & $\begin{array}{l}\text { F: AACAACCAGGAGGAAAGTAA } \\
\text { R: CTGATCAAGTCGCAAAGC }\end{array}$ \\
\hline envR & erv3 & HERV-R (HERV3) & 100 & Chr7: 63863079-63865094 (-) & $\begin{array}{l}\text { F: GGTTAGAAATCTGAAGTCC } \\
\text { R: AAAGTCAATGACAGATGCGG }\end{array}$ \\
\hline envR(b) & - & HERV-R(b) (PABL_B) & 50 & Chr3: |67868|4-16788358 (+) & $\begin{array}{l}\text { F: GCTAAGCACCAGTTCAGCACTG } \\
\text { R: TGTTTTGGGACACCACGAAT }\end{array}$ \\
\hline envFRD & Syncytin 2 & HERV-FRD (MER50) & 3000 & Chr:6: II211913-11213529(-) & $\begin{array}{l}\text { F: CTTGTACACCACCAGGAGTTCC } \\
\text { R: TTTGAGCAAGGGTGATTCAT }\end{array}$ \\
\hline
\end{tabular}

ing $e n v \mathrm{FRD}, e n v \mathrm{~W}, e n v \mathrm{~F}(\mathrm{c}) 1, e n v \mathrm{H} 1$ and $e n v \mathrm{H} 3)$, it ranges from 0.11 for $e n v \mathrm{R}(\mathrm{b})$ to 0.66 for $e n v \mathrm{~T}$, close to the pseudogene ratio.

Based on the number of nonsynonymous and indel mutations, a hierarchy among the endogenous coding env genes can be established, with the envW, envFRD and env $\mathrm{F}(\mathrm{c}) 1$ genes being the most conserved (see also [19]), and the $e n v \mathrm{~K} 1$, $e n v \mathrm{~K} 2, e n v \mathrm{~T}$, $e n v \mathrm{R}$, $e n v \mathrm{R}(\mathrm{b})$, and $e n v \mathrm{~F}(\mathrm{c}) 2$ genes being affected by numerous mutations, including mutations resulting in truncation of the protein due to frameshifting or generation of stop codons. There is no correlation between the number of SNPs and the "age" of the corresponding gene in the primate lineage. This is clearly illustrated in Figure 3, where the env genes have been ordered according to the date of entry of each corresponding provirus into the host genome as previously determined via an analysis of the orthologous loci throughout evolution (reviewed in [4]). For instance, the $e n v$ genes of the HERV-K(HML-2) family are human-specific, i.e. are present in the genome of primate since less than five million years (Myrs), whereas envR(b) and $e n v$ FRD have entered the genome of the common ancestor of Old World and New World monkeys more than 40 Myrs ago. For the envFRD gene, which is among the "oldest" env genes, only 4 SNPs are found, whereas for the "recent" env genes of the HERV-K(HML-2) family, the SNP number can be as high as 16. Although the lack of correlation between the "age" of the genes in the primate lineage and the numbers of SNPs is not unexpected taking into account the occurrence of "bottlenecks" giving rise to founder effects during the evolution of the human population, what remains surprising is the important variability of the number and "severity" of SNPs among the env genes. This should be a strong indication for a differential selection pressure exerted on these genes (see below).

A further characterization of the SNPs, including genotype distribution, haplotype frequency, and linkage disequilibrium was performed (additional data files 1,2 and 3). The genotype distributions are compatible with a HardyWeinberg equilibrium, except for some positions on the HERV-K1 (HERV-K1_44, HERV-K1_133, HERV-K1_403, HERV-K1_482, HERV-K1_651， HERV-K1_673， HERVK1_2144), HERV-K4 (HERV-K4_292, HERV-K4_369, HERV-K4_382, HERV-K4_586) and HERV-F(c)1 (HERV$\mathrm{F}(\mathrm{c}) 1$ 122, HERV-F(c)1_226, HERV-F(c)1_235) env genes, consistent with recent integration of these elements in the primate lineage (see Figure 3). Haplotype frequencies were estimated for each gene based on the Expectation-Maximization (EM) algorithm [20] for haplotypes with frequency estimates $>1 \%$. The results are summa- 


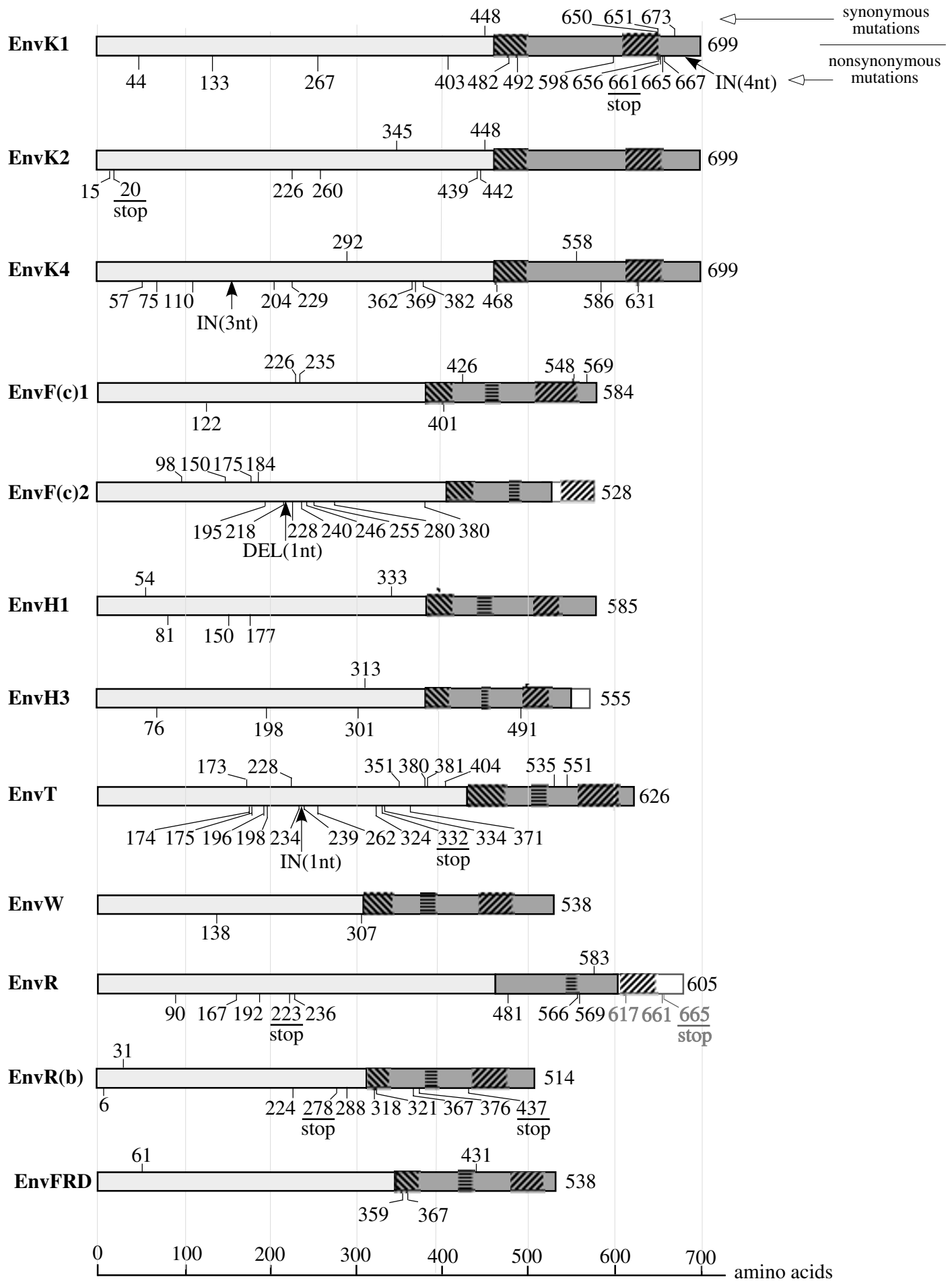

\section{Figure 2}

Localization of the SNPs on the envelope genes. Characteristic domains of the envelope proteins are depicted as in Figure IB. The gray frames at the end of $H 3, R$ and $F(c) 2$ genes represent short open reading frames present downstream of the stop codon. Mutations are represented along the protein sequence, with the number corresponding to the amino acid affected by the SNP. Synonymous mutations are indicated above the protein frame. Non-synonymous (with stop mutations indicated) and indel mutations (with frame shifting mutations underlined) are indicated below the protein frame. 


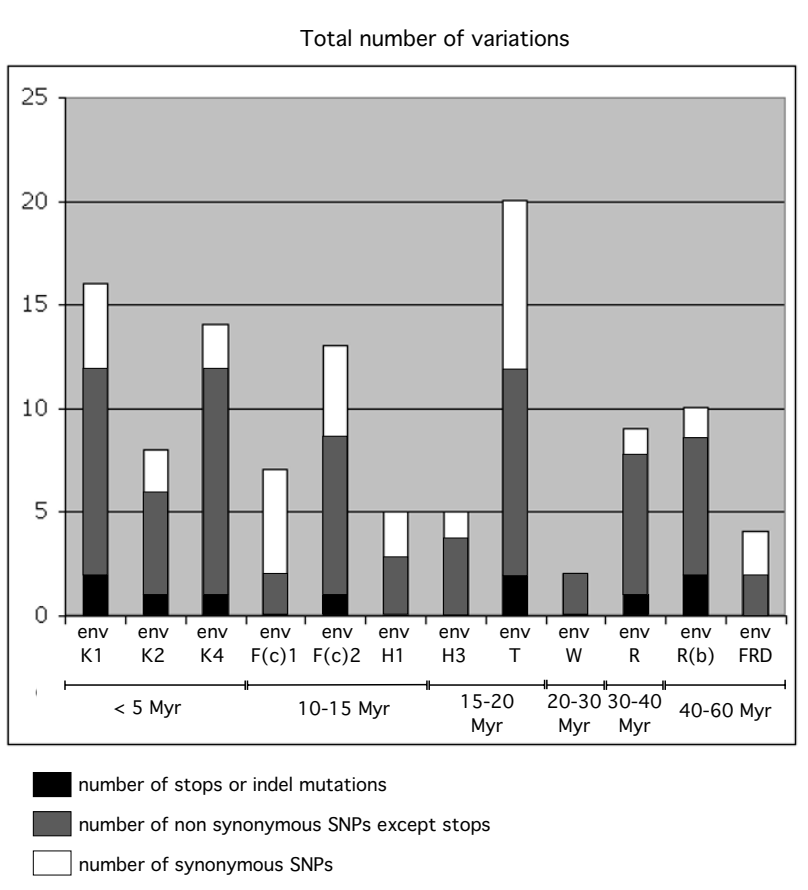

Figure 3

Number of variations identified in the HERV coding envelope genes. The SNP numbers are indicated for each env gene, with the number of synonymous SNPs, nonsynonymous SNPs except stops, and stops or indel mutations indicated. The env genes are ordered according to their date of entry in the primate lineage (see [4]), indicated in million years (Myr).

rized in additional data file 2 . The three most frequent haplotypes for each env gene represent $>80 \%$ of all the haplotypes, suggesting that these regions have a low recombination rate. A linkage disequilibrium (LD) plot was generated (additional data file 3 ) with pairwise LDs measured between each pair of polymorphisms using the D and D' methods (see materials and methods). As expected, the majority of high $\mathrm{LD}$ values occur within the env genes, and the LDs calculated between SNPs of different HERV env genes is low. Low LDs were obtained as well for $e n v$ genes located on the same chromosome, i.e. for HERV-F(c)2, HERV-K2, HERV-R, HERV-W (on chromosome 7) and for HERV-H1 and HERV-H3 (on chromosome 2; $10 \mathrm{Mb}$ apart). These observations suggest an independent evolution for each $e n v$ gene.

Among all the coding $e n v$ genes, $e n v \mathrm{~W}$ and $e n v \mathrm{FRD}$ are the only two genes with a clearly identified functional property, i.e. the capacity to generate cell-cell fusion $[10,12]$. This property - most probably associated with its role in placentation, see the Background section - was used to characterize further the consequences of the identified SNPs on the fusogenic function of the encoded proteins. To do so, we PCR-amplified the genomic DNAs of individuals carrying the corresponding SNP alleles, with primers allowing the cloning of the env genes in appropriate expression vectors. The fusogenic function was then assayed as in [12], using two different cell lines for fusion (Figure 4). As illustrated in the figure, no difference can be observed between the four haplotypes of each of the env genes tested. This, together with the low SNP level for the two genes, is a strong indication for selection of a "function" associated with the corresponding proteins.

\section{Interspecific sequence conservation}

The data regarding the orthologous genes that can be found in primates for each of the presently studied human env genes are given in Figure 5. Some of the orthologous primate env genes had been previously cloned and sequenced ([19] for $e n v \mathrm{~W}$, [21] for $e n v \mathrm{FRD}$, [22] for $e n v \mathrm{R}$ and [23] for envF(c)1). Others have been PCR-amplified and tested using a direct coupled in vitro transcription/ translation assay to determine their coding status ([24] for the $3 e n v \mathrm{H}$ and this study for envT, envF(c)2 and envR(b), see Materials and Methods). As illustrated in the figure, a first important outcome is that only three env genes have been maintained in a fully coding state throughout evolution, i.e. envFRD (7/7 lineages), env $\mathrm{W}$ (5/5 lineages) and $e n v \mathrm{R}$ (5/5 lineages) which, interestingly, correspond to the three env genes highly expressed in the placenta. Secondly, as observed for the human SNP analysis, there is no correlation between the "age" of the gene in the primate lineage and its coding status: the latter three env genes are among the "oldest" ones, whereas three other env genes $(e n v \mathrm{H} 3$, env $\mathrm{T}$ and $e n v \mathrm{~F}(\mathrm{c}) 1)$ are fully coding in only one non-human lineage, and the others are only coding in the human lineage.

\section{Discussion}

The present investigation of the fully coding human env genes, including the human SNP search and the analysis of the coding status of the identified primate orthologs, pinpoints two of these genes - namely envFRD and envW - that disclose the characteristic features of a gene subjected to a functional constraint, i.e. low polymorphism and maintenance of an open reading frame during evolution. Interestingly, these two genes are highly - and specifically - expressed in the placenta, and possess a wellcharacterized fusogenic function which led to the proposal that they are bona fide genes that have been co-opted by the host for a physiological function related to placenta physiology $[10,12,13,19]$. Among the other genes, env $\mathrm{R}$ is of interest since as with the two former genes it is highly expressed in the placenta and has maintained its fully coding status in all species since its entry into the primate lineage. Yet, the SNP analysis discloses a severe 
A

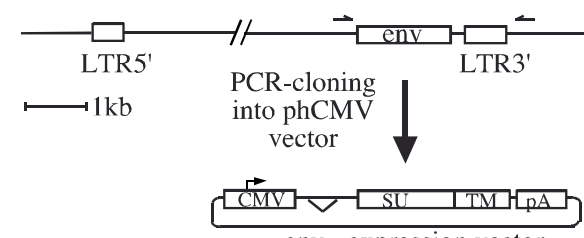

env - expression vector

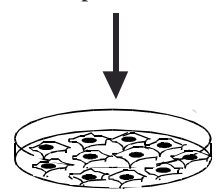

target cells

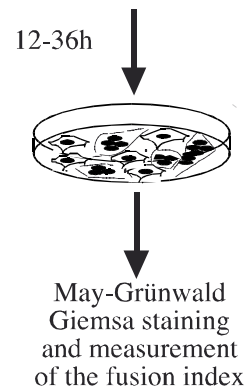

B
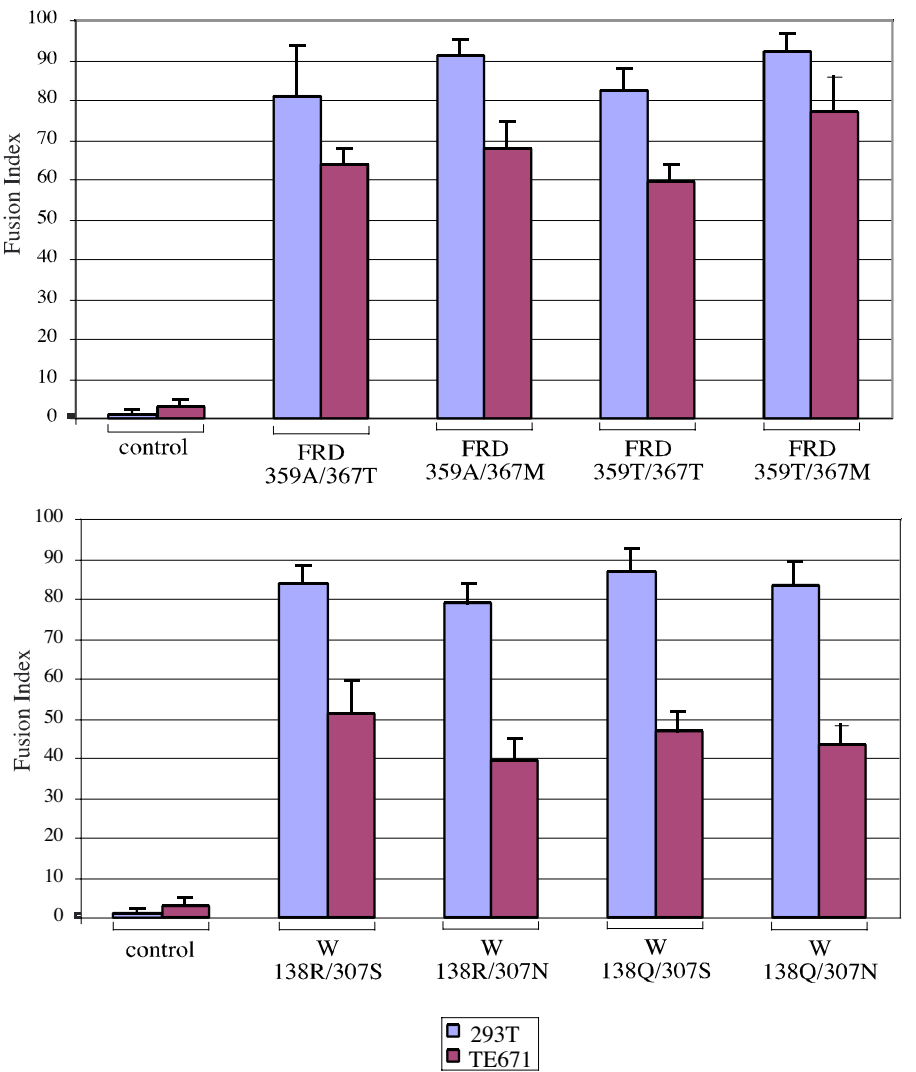

\section{Figure 4}

Effects of the non synonymous SNPs identified in envFRD and envW on their fusogenic function. A, Construction of the env-expression vectors and rationale of the fusion assay. Each of the env allelic forms were PCR amplified from genomic DNA and cloned into the phCMV expression vector. Cells were transfected with the env-expression vectors and stained with May-Grünwald and Giemsa solutions. B, Cell-cell fusion assay for the allelic forms of envFRD (upper panel) and envW (lower panel), using two cells types (human 293T and TE67I cells). The fusion index represents the percentage of fusion events in the transfected cell populations as evidenced by syncytia formation, and is quantitated as in [12]. The control corresponds to transfection of an expression vector without an env gene. The most frequent haplotypes correspond to FRD359A/367T and WI38R/307S.

polymorphism - including a premature stop codon indicating that the preservation of an open reading frame during evolution should not be considered as a sufficient criterion to assign a biological function. The other genes are not conserved in a fully coding state in all the primate branches where they are present and/or disclose a severe polymorphism (with in several cases occurrence of a premature stop codon for some allelic forms).

Another hallmark of the presence of a functional constraint on a gene is a low nonsynonymous/synonymous substitution ratio $(\mathrm{Ka} / \mathrm{Ks})$ for orthologous genes found in other species. A bona fide gene with a cellular function should be under purifying selection, which prevents deleterious nonsynonymous substitutions from being fixed and usually does not affect synonymous substitutions, leading to $\mathrm{Ka} / \mathrm{Ks}$ ratios $<1$, whereas a gene under genetic drift (e.g. a pseudogene) has a Ka/Ks ratio close to unity (reviewed in [25]). Such an analysis has already been performed for the envFRD and the env $\mathrm{W}$ genes. The mean ratio for all pairwise comparisons was 0.37 for envFRD [21], demonstrating the existence of a selective pressure. For $e n v \mathrm{~W}$, the corresponding ratio was 0.8 [19], precluding definite conclusions (yet, a subsequent study on envW identified a region of the gene with a lower ratio compatible with a functional constraint specifically exerted on 


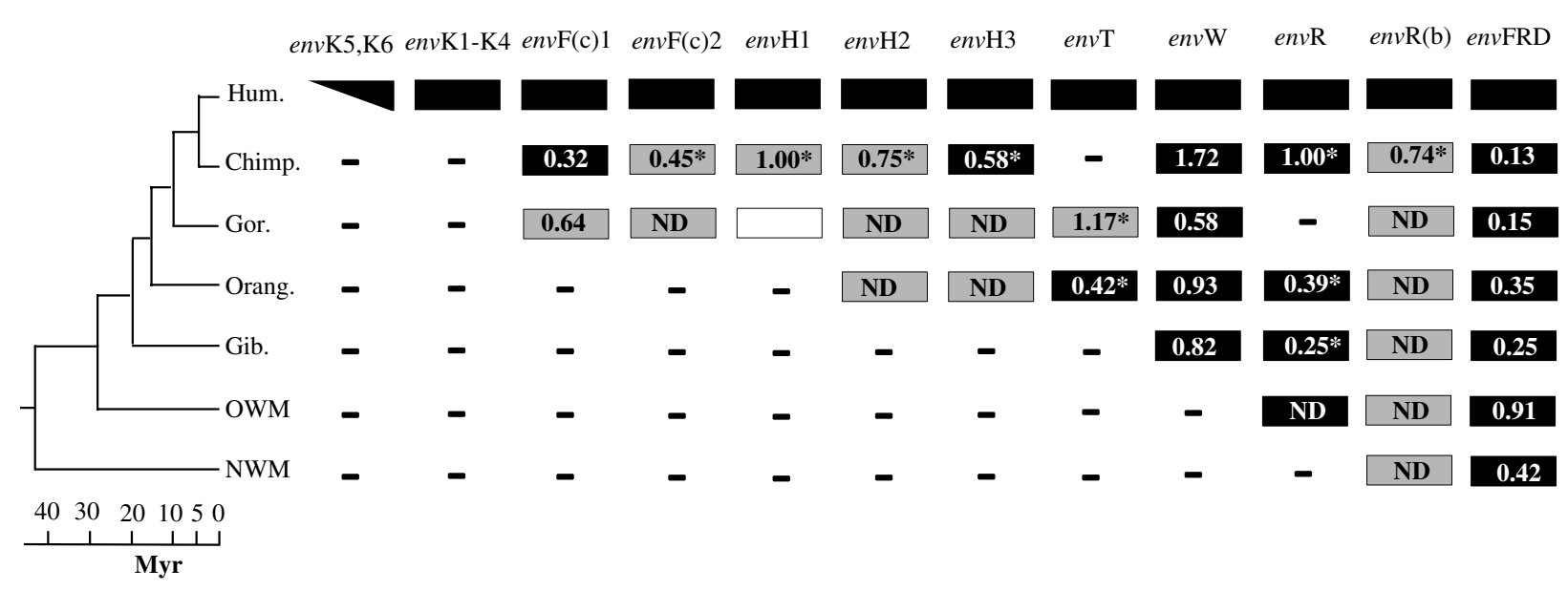

0.32 coding envelope (Ka/Ks ratio between human and the corresponding primate)

$\square$ present but non coding envelope
absent envelope (provirus as a solo LTR)
provirus present in a fraction of the human population with a coding envelope
absent provirus

\section{Figure 5}

Conservation of the coding envelopes in primate species. Hum.: human, Chimp.: chimpanzee, Gor.: gorilla, Orang.: orangutan, Gib.: gibbon, OWM: Old World Monkeys, NWM: New World Monkeys. The boxes indicate presence of the env gene, with its coding status illustrated with a color code.

that domain [26]). We have calculated the Ka/Ks ratios for the other env genes, when nucleotide sequences of their orthologs were available (data not shown). These ratios were found to be heterogeneous for a given env gene, with values ranging from 0.23 to 1.17 , again precluding any definite conclusion to be drawn.

According to the present analysis of intra- and interspecific variability of the env genes, one is led to conclude that most probably only two among the twelve studied env genes are likely to be involved in an essential human physiological function, whereas the others would be on their way to conversion into pseudogenes. The presence of a reading frame still open in human for the latter genes may appear intriguing, but one has to keep in mind that they belong to multicopy HERV families, and as such one of the element could have remained open just by chance, without any purifying selection, since even under completely neutral drift it takes time for a sufficient number of mutations to transform a gene into a pseudogene. Along this line, it is of interest to mention the study by Zhang and Webb on the primate $V 1 R$ pheromone receptor genes, for which there were approximately 140 copies in the genome of the common ancestor of Old World monkeys and hominoids, whereas the human genome has only five $V 1 R$ genes that retained an ORF. Examination of the orthologous genes in primates showed that none of the five genes kept an intact ORF in all of the apes. Furthermore, for the orthologous sequences with an intact ORF, $\mathrm{Ka} / \mathrm{Ks}$ ratios were close to unity. The intraspecific variation of these five human genes was also assessed, and for two of them an allelic form generating a premature stop codon was found. Altogether, the authors concluded that there were no functional constraints on these genes since before the separation of hominoids and Old World Monkeys (approximately 23 Myrs ago) and that they were in the process of pseudogeneization in those primate species [27]. Another possible explanation for the "neutral" conservation of an open reading frame for an HERV env gene without any selection pressure from the host could be related to the relatively autonomous status of these parasitic elements, and be associated with the persistence of active retroviral elements responsible for the maintenance 
- by a reiterated infection process - of some of the HERV families (e.g. $[23,28])$.

In any case, it appears clearly that conservation of an env gene with a coding status cannot be taken as the sole criterion for a possible function to the benefit of the host, with only the env $\mathrm{W}$ and $e n v \mathrm{FRD}$ genes emerging from the present study as possible bona fide genes. Yet, this does not mean that the other $e n v$ genes cannot have any effect in humans. Indeed, the present analysis only indicates that they are not under stringent purifying selection, in terms of evolution, but they still could be involved in pathologies - such as tumors or auto-immune diseases - not deleterious to the species because they occur late in the life of the individuals. One should keep in mind that endogenous retroviruses originate from bona fide retroviruses, and as such might have conserved some of the pathological potency of their progenitors. In this respect, the identified SNPs should be essential tools to determine if this is indeed the case, via an analysis of their distribution among selected groups of individuals with a definite pathology. Along this line, the present data on the envT gene are of special interest. Indeed, this gene is the only non-placental env gene found to be highly expressed in a human tissue - the thyroid - of healthy individuals [6], and the high level of polymorphism of the gene shown in this report together with its lack of conservation in primates suggest that it is not involved in any essential physiological process and thus not subjected to purifying selection. Thanks to the identified SNPs, it can now be tested whether this expressed gene is involved in a pathological process in humans, among which thyroid tumors could be select candidates for a systematic search.

\section{Conclusion}

The systematic SNP search on fully coding human endogenous envelope genes, combined with an analysis of the sequence conservation among the orthologs that can be identified among primates revealed that two genes (env $\mathrm{W}$ and envFRD) can be considered as bona fide genes, and identified polymorphisms - to a variable extent - in the other genes. The data are consistent with a physiological role for the former (also called syncytin-1 and syncytin-2 and likely to be involved in human placentation) and provide tools for the latter, to determine their potential role in physiological processes and/or their association with pathological processes in humans - which would be the consequence of their original retroviral status.

\section{Methods}

\section{DNA samples and genotyping}

Ninety-one human samples of French Caucasians were collected from the EGEA (Epidemiological study on the Genetics and Environment of Asthma) study, among the controls ascertained without disease. PCR was performed in mixture containing $25 \mathrm{ng}$ of DNA, $0.3 \mathrm{pmol}$ of each primer, $6 \mathrm{nmol}$ of each dNTP, 0.75 units of ExTaq and 1× reaction buffer (Takara). Sequencing reactions were performed according to the Dye Terminator method using an ABI PRISM ${ }^{\circledast} 3700$ DNA Analyzer (Applied Biosystems, Foster City, CA, USA). Alignment of sequences, SNP discovery and genotyping were performed with Genalys software [29]. The genomic sequences used for the alignment are envFRD (GenBank accession no. AL136139), envR

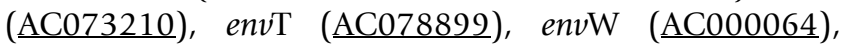
$e n v \mathrm{Fc}(1) \quad(\underline{\mathrm{AL} 354685}), \quad e n v \mathrm{Fc}(2) \quad(\underline{\mathrm{AC} 01222}), \quad e n v \mathrm{H} 1$

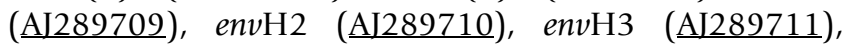
$e n v \mathrm{R}(\mathrm{b}) \quad(\underline{\mathrm{AC} 093488}), \quad e n v \mathrm{~K} 1 \quad(\underline{\mathrm{AC} 074261}), \quad e n v \mathrm{~K} 2$ (AC072054), envK4 (트164615). The sources of the simian genomic DNAs are given in ref [24].

\section{Statistical analysis}

The haplotypes frequencies using all polymorphisms for each gene were estimated with the EM Algorithm [20]. The linkage disequilibrium (LD) estimates between pairs of polymorphisms were obtained by estimating the two polymorphisms haplotypes frequencies using this algorithm. Computation of $\mathrm{D}$ and $\mathrm{D}^{\prime}$ (standard disequilibrium measure and standardized disequilibrium measure) in additional data file 3 was performed as in ref [30].

\section{Cloning of allelic forms of the $W$ and FRD human env genes in expression vectors}

The FRD and $\mathrm{W}$ env genes were PCR-amplified from human genomic DNAs. PCR was carried out for 25 cycles $\left(10 \mathrm{sec}\right.$ at $93^{\circ} \mathrm{C}, 30 \mathrm{sec}$ at $56^{\circ} \mathrm{C}, 4 \mathrm{~min}$ at $68^{\circ} \mathrm{C}$ ), in $50 \mu \mathrm{l}$, using 100 ng of genomic DNA, 48 pmol of each primer, $350 \mu \mathrm{M}$ of each $\mathrm{dNTP}, 0.75 \mu \mathrm{l}$ Expand long template enzyme mix and $1 \times$ reaction buffer (Roche Applied Science). For the FRD env gene, Xhol-containing primers were ATCACCTCGAGCACCATGGGCCTGCTCCTGCTGGT-

TCTCATTC as forward primer and ATCACCTCGAGGCTTCAGTACAGGTGGATA as reverse primer. For the $\mathrm{W}$ env gene, Xhol-containing primers were ATCACCTCGAGAACAACCAGGAGGAAAGTAA as forward primer and ATCACCTCGAGCTGATCAAGTCGCAAAGC as reverse primer. Each PCR product was then XhoI-cleaved and cloned into the phCMV-G vector (described in [12]) opened with XhoI. Allelic forms of the cloned env genes were assessed by enzymatic restriction. For envFRD, the 1075 G->A transition (aa 359) predicts the loss of a RsaI site and the $1100 \mathrm{C}->\mathrm{T}$ transition (aa 367) predicts the loss of a BstUI site, and for $e n v \mathrm{~W}$ the $413 \mathrm{G}->\mathrm{A}$ transition (aa 138) predicts the gain of a BstXI site and the $920 \mathrm{G}->\mathrm{A}$ transition (aa 307) predicts the gain of a Tsp509I site. As two allelic forms (FRD359T/367M and W138Q/307S) were not available among the cloned envelope genes, we constructed them by exchange of restriction fragments (BsmI-NotI for FRD359T/367M and KpnI-XhoI for W138Q/307S). 


\section{Cell-cell fusion assay}

The human TE671 rhabdomyosarcoma cells (ATCC CRL8805) and 293T embryonal kidney cells (ATCC CRL11268) were grown in Dulbeco's modified Eagle's medium (DMEM) supplemented with 10\% fetal calf serum. All cell culture media were supplemented with streptomycin $(100 \mu \mathrm{g} / \mathrm{mL})$ and penicillin $(100 \mathrm{U} / \mathrm{mL})$. Cells were transfected using calcium phosphate precipitation (Invitrogen, $5 \mu \mathrm{g}$ of DNA for $5 \times 10^{5}$ cells). Fusion activity of envelope glycoproteins was measured 12 to 36 $\mathrm{h}$ after transfection of the cells with the corresponding expression vectors. To visualize syncytia, cells were fixed in methanol and stained by adding May-Grünwald and Giemsa solutions (Sigma) according to the manufacturer's instructions. The fusion index, which represents the percentage of fusion events in a cell population is defined as $[(\mathrm{N}-\mathrm{S}) / \mathrm{T}] \times 100$, where $\mathrm{N}$ is the number of nuclei in the syncytia, $\mathrm{S}$ is the number of syncytia, and $\mathrm{T}$ is the total number of nuclei counted.

\section{Characterization of the orthologous envT, envF(c)2 and envR(b) env gene ORFs from simians}

The size of the env gene open reading frame in the primate loci was evaluated using a direct coupled in vitro transcription/translation assay based on $\mathrm{T} 7$ promoter-containing PCR products as described in [24], which allows to determine the status of both alleles in the same assay.

For the amplification of $e n v \mathrm{~T}$ from gorilla and orangutan, the forward $\mathrm{T} 7$ promoter-containing primers were GCTAATACGACTCACTATAGGAACAGACCACCATGTCCTGCTTGGATTCATCAC and GCTAATACGACTCACTATAGGAACAGACCACCATGTTGGATTCATCACTCCCA, respectively, and the common reverse flanking primer was CTGAAGGGAGTTCCTCCTAGG. For the amplification of $e n v$ R(b) from chimpanzee, gorilla, orangutan, gibbon, Rhesus macaque (Old World Monkey) and Callithrix jacchus (New World Monkey) the forward $\mathrm{T} 7$ promoter-containing primer was GCTAATACGACTCACTATAGGAACAGACCACCAT-

GGATCCACTACACACGATTGA and the reverse flanking primer was TGTTTTGGGACACCACGAAT. For the amplification of $e n v \mathrm{~F}(\mathrm{c}) 2$ from chimpanzee and gorilla, the forward $\mathrm{T} 7$ promoter-containing primer was GCTAATACGACTCACTATAGGAACAGACCACCAT-

GAATTCTCCATGTGAC and the reverse flanking primer was GACACTTAATAGTTGCGACA.

The simian env gene sequences were deposited in Genbank with accession numbers AJ862646-AJ862655.

\section{List of abbreviations used}

HERV, human endogenous retrovirus; TM, transmembrane; SNP, single nucleotide polymorphism; LTR, Long Terminal Repeat; LD, linkage disequilibrium.

\section{Authors' contributions}

$\mathrm{NdP}$ designed the cloning primers for the env genes, carried out the interspecific sequence conservation studies and drafted the manuscript.

GD carried out the SNP studies.

SB carried out the cloning of the env genes and the cell-cell fusion assays.

FH participated in the interspecific sequence conservation studies.

AV designed the sequencing primers, participated in the coordination of the SNP studies and in drafting the manuscript.

FM coordinated the SNP studies.

TH conceived the study.

\section{Additional material}

\section{Additional data file 1}

Additional data file 1 is a table listing the polymorphism of HERV coding envelope genes.

Click here for file

[http://www.biomedcentral.com/content/supplementary/1471-

2164-6-117-S1.DOC]

\section{Additional data file 2}

Additional data file 2 is a figure showing polymorphism combinations and estimated frequencies for haplotypes in the 12 HERV coding env genes. The SNPs are identified by their CNG ID (see additional data file 1). Click here for file

[http://www.biomedcentral.com/content/supplementary/14712164-6-117-S2.PDF]

\section{Additional data file 3}

Additional data file 3 is a figure showing the LD plot of 12 HERV coding env genes. The LD pattern is shown with the $D$ values above and the $D^{\prime}$ values below the diagonal, and estimated allele frequencies for each polymorphism. Different colors are used to represent ranges of positive D and $D^{\prime}$ values. The SNPs are identified by their CNG ID (see additional data file 1).

Click here for file

[http://www.biomedcentral.com/content/supplementary/1471-

2164-6-117-S3.PDF]

\section{Acknowledgements}

This work was supported by the CNRS and by grants from the Ligue Nationale contre le Cancer (Equipe Labellisée for T.H.). We thank Evelyne Heyer for helpful discussions and acknowledge Christian Lavialle for critical reading of the manuscript. 


\section{References}

I. Consortium IHGS: Initial sequencing and analysis of the human genome. Nature 2001, 409:860-921.

2. Boeke JD, Stoye JP: Retrotransposons, endogenous retroviruses, and the evolution of retroelements. In Retroviruses Edited by: Coffin JM, Hughes SH and Varmus HE. New York, Cold Spring Harbor Laboratory Press; 1997:343-436.

3. Bannert N, Kurth R: Retroelements and the human genome: New perspectives on an old relation. Proceedings of the National Academy of Sciences of the United States of America 2004, I 3 Supp 2: |4572-14579.

4. de Parseval N, Heidmann T: Human endogenous retroviruses: from infectious elements to human genes. Cytogenet Genome Res 2005, I 1 0:318-332.

5. Repbase update, a database of transposable elements [http:/ /www.girinst.org]

6. de Parseval N, Lazar V, Casella JF, Benit L, Heidmann T: Survey of human genes of retroviral origin: identification and transcriptome of the genes with coding capacity for complete envelope proteins. Journal of Virology 2003, 77: I04 |4- 10422.

7. Best S, Le Tissier PR, Stoye JP: Endogenous retroviruses and the evolution of resistance to retroviral infection. Trends in Microbiology 1997, 5:313-318.

8. Cianciolo G], Copeland T, Orozlan S, Snyderman R: Inhibition of lymphocyte proliferation by a synthetic peptide homologous to retroviral envelope protein. Science 1985, 230:453-455.

9. Mangeney $M$, Heidmann T: Tumor cells expressing a retrovira envelope escape immune rejection in vivo. Proc Natl Acad $\mathrm{SC}$ USA 1998, 95:|4920-|4925.

10. Blond JL, Lavillette D, Cheynet V, Bouton O, Oriol G, Chapel-Fernandes S, Mandrand B, Mallet F, Cosset FL: An envelope glycoprotein of the human endogenous retrovirus HERV-W is expressed in the human placenta and fuses cells expressing the type D mammalian retrovirus receptor. Journal of Virology 2000, 74:3321-3329.

II. Mi S, Lee X, Li X, Veldman GM, Finnerty H, Racie L, LaVallie E, Tang $X Y$, Edouard P, Howes S, Keith JCJ, McCoy JM: Syncytin is a captive retroviral envelope protein involved in human placental morphogenesis. Nature 2000, 403:785-788.

12. Blaise S, de Parseval N, Bénit L, Heidmann T: Genomewide screening for fusogenic human endogenous retrovirus envelopes identifies syncytin 2 , a gene conserved on primate evolution. Proceedings of the National Academy of Sciences of the United States of America 2003, 100:13013-13018.

13. Frendo JL, Olivier D, Cheynet V, Blond JL, Bouton O, Vidaud M, Rabreau $M$, Evain-Brion $D$, Mallet F: Direct involvement of HERV-W Env glycoprotein in human trophoblast cell fusion and differentiation. Mol Cell Biol 2003, 23:3566-3574.

14. de Parseval N, Heidmann T: Physiological knock-out of the envelope gene of the single copy ERV-3 human endogenous retrovirus in a fraction of the caucasian population. Journal of Virology 1998, 72:3442-3445.

15. Turner G, Barbulescu M, Su M, Jensen-Seaman MI, Kidd KK, Lenz J: Insertional polymorphisms of full-length endogenous retroviruses in humans. Current Biology 200I, I I: I53 I-I535.

16. Hughes JF, Coffin JM: Human endogenous retrovirus $K$ soloLTR formation and insertional polymorphisms: implications for human and viral evolution. Proc Natl Acad Sci U S A 2004, I01:1668-1672.

17. Zhao Z, Fu YX, Hewett-Emmett D, Boerwinkle E: Investigating single nucleotide polymorphism (SNP) density in the human genome and its implications for molecular evolution. Gene 2003, 3 I 2:207-2 I3

18. Stephens JC, Schneider JA, Tanguay DA, Choi J, Acharya T, Stanley SE, Jiang R, Messer CJ, Chew A, Han JH, Duan J, Carr JL, Lee MS, Koshy B, Kumar AM, Zhang G, Newell WR, Windemuth A, Xu C, Kalbfleisch TS, Shaner SL, Arnold K, Schulz V, Drysdale CM, Nandabalan K, Judson RS, Ruano G, Vovis GF: Haplotype variation and linkage disequilibrium in 3 I 3 human genes. Science 200 I, 293:489-93. Epub 2001 Jul 12.

19. Mallet F, Bouton O, Prudhomme S, Cheynet V, Oriol G, Bonnaud B, Lucotte $G$, Duret $L$, Mandrand $B$ : The endogenous retroviral locus ERVWEI is a bona fide gene involved in hominoid placental physiology. Proc Natl Acad Sci U S A 2004, I 0 I: |73 |-I736.
20. Laird N: Computational Statistic: the EM algorithm. In Handbook of Statistics Volume 9. Edited by: Rao CR. Amsterdam, Elsevier Science Publishers; 1993:509-520.

21. Blaise S, Ruggieri A, Dewannieux M, Cosset FL, Heidmann T: Identification of an envelope protein from the FRD family of Human Endogenous Retroviruses (HERV-FRD) conferring infectivity on retroviral particles and functional conservation among simians. Journal of Virology 2004, 78: 1050- 1054.

22. Herve CA, Forrest G, Lower R, Griffiths DJ, Venables PJ: Conservation and loss of the ERV3 open reading frame in primates. Genomics 2004, 83:940-943.

23. Benit L, Calteau A, Heidmann T: Characterization of the lowcopy HERV-Fc family: evidence for recent integrations in primates of elements with coding envelope genes. Virology 2003, 312:159-168.

24. de Parseval N, Casella JF, Gressin L, Heidmann T: Characterization of the three HERV-H proviruses with an open envelope reading frame encompassing the immunosuppressive domain and evolutionary history in primates. Virology 2001, 279:558-569.

25. Fay JC, Wu Cl: Sequence divergence, functional constraint, and selection in protein evolution. Annu Rev Genomics Hum Genet 2003, 4:213-235.

26. Bonnaud B, Bouton O, Oriol G, Cheynet V, Duret L, Mallet F: Evidence of selection on the domesticated ERVWEI env retroviral element involved in placentation. Mol Biol Evol 2004, 2 I: |895-1901.

27. Zhang J, Webb DM: Evolutionary deterioration of the vomeronasal pheromone transduction pathway in catarrhine primates. Proc Natl Acad Sci U S A 2003, I 00:8337-834I.

28. Belshaw R, Pereira V, Katzourakis A, Talbot G, Paces J, Burt A, Tristem $M$ : Long-term reinfection of the human genome by endogenous retroviruses. Proc Natl Acad Sci U S A 2004, I $01: 4894-4899$

29. Takahashi M, Matsuda F, Margetic N, Lathrop M: Automated identification of single nucleotide polymorphisms from sequencing data. J Bioinform Comput Biol 2003, I:253-265.

30. Devlin B, Risch N: A comparison of linkage disequilibrium measures for fine-scale mapping. Genomics 1995, 29:3 I I-322.

Publish with Bio Med Central and every scientist can read your work free of charge

"BioMed Central will be the most significant development for disseminating the results of biomedical research in our lifetime. "

Sir Paul Nurse, Cancer Research UK

Your research papers will be:

- available free of charge to the entire biomedical community

- peer reviewed and published immediately upon acceptance

- cited in PubMed and archived on PubMed Central

- yours - you keep the copyright
BiolMedcentral 